PROCEEDINGS OF THE

AMERICAN MATHEMATICAL SOCIETY

Volume 128, Number 8, Pages 2479-2485

S 0002-9939(00)05288-6

Article electronically published on February 25, 2000

\title{
ON ROUGHLY TRANSITIVE AMENABLE GRAPHS AND HARMONIC DIRICHLET FUNCTIONS
}

\author{
GÁBOR ELEK AND GÁBOR TARDOS
}

(Communicated by Józef Dodziuk)

\begin{abstract}
We introduce the notion of rough transitivity and prove that there exist no non-constant harmonic Dirichlet functions on amenable roughly transitive graphs.
\end{abstract}

\section{INTRODUCTION}

Let us call a graph $G$ roughly transitive if there exist quasi-isometries $f_{x y}$ from $G$ to $G$ for vertices $x$ and $y$ of $G$ with $f_{x y}(x)=y$ such that these quasi-isometries have bounded distortion. (See Section 2 for notation.)

Any graph quasi-isometric to a vertex-transitive graph is clearly roughly transitive. However, the converse is far from being true as we shall show in Section 3.

The study of harmonic Dirichlet functions on graphs goes back to Cheeger and Gromov [2]. They proved that there exist no non-constant harmonic Dirichlet functions on the Cayley graph of an amenable group. For different proofs see also Elek [3] and Paschke [7]. Later Medolla and Soardi [5] extended this result to amenable vertex-transitive graphs. In a recent preprint Benjamini, Lyons, Peres, and Schramm [1] gave a probabilistic proof for this result. The goal of this paper is to extend the result of Medolla and Soardi to amenable roughly transitive graphs:

Theorem 1. The only harmonic Dirichlet functions on a roughly transitive amenable graph are the constant functions.

Note that some assumption of this kind is necessary as there exist amenable graphs with non-constant harmonic Dirichlet functions (see e.g. [10, Chapter 6).

\section{Notation}

In this note we use the term graph for simple, connected, undirected graphs with bounded degree vertices only. We will mostly consider infinite graphs. We denote the set of vertices of a graph $G$ by $V(G)$; we use $E(G)$ for the set of edges. By $\vec{E}(G)$ we denote the set of oriented edges: $\vec{E}(G)=\{(x, y) \mid\{x, y\} \in E(G)\}$. We denote the opposite orientation $(y, x)$ of an oriented edge $e=(x, y)$ by $\bar{e}$. We consider

Received by the editors July 6, 1998 and, in revised form, September 8, 1998 .

1991 Mathematics Subject Classification. Primary 58G05.

Key words and phrases. Amenable graphs, harmonic functions.

The first author was supported by OTKA grant T25004 and the Bolyai Fellowship.

The second author was supported by OTKA grants F014919, T029255 and AKP grant 97-56. 
the Hilbert space $l_{2}(G)$ of the $l_{2}$ functions $u: \vec{E}(G) \rightarrow \mathbb{R}$ satisfying $u(\bar{e})=-u(e)$ with the scalar product $\left\langle u, u^{\prime}\right\rangle=1 / 2 \sum_{e \in \vec{E}(G)} u(e) u^{\prime}(e)$. For simplicity we write $u(x, y)$ for the value $u((x, y))$. For a function $v: V(G) \rightarrow \mathbb{R}$ we define its differential $d v: \vec{E}(G) \rightarrow \mathbb{R}$ by $d v(x, y)=v(y)-v(x)$. We call a $v: V(G) \rightarrow \mathbb{R}$ function a Dirichlet function if $d v \in l_{2}(G)$ and denote the set of Dirichlet functions by $D(G)$. Let $l_{2}\left(V(G)\right.$ ) denote the Hilbert space of the $l_{2}$ functions $v: V(G) \rightarrow \mathbb{R}$ (with the standard scalar product); this is clearly contained in $D(G)$.

Consider the adjoint $d^{*}$ of the operator $d: l_{2}(V(G)) \rightarrow l_{2}(G)$. We call a function $u \in l_{2}(G)$ a flow if $d^{*} u=0$. We call a Dirichlet function $v \in D(G)$ harmonic if $d v$ is a flow. We denote the set of harmonic Dirichlet functions by $H D(G)$.

Here $d^{*} u$ is given by $d^{*} u(x)=\sum_{\{y, x\} \in E(G)} u(y, x)$ for $u \in l_{2}(G)$ and $x \in V(G)$. Thus $u$ is a flow if and only if $\sum_{\{x, y\} \in E(G)} u(x, y)=0$ for every $x \in V$. The function $v \in D(G)$ is harmonic if and only if for every $x \in V$ the value $v(x)$ is the average of the values $v(y)$ with $\{x, y\} \in E(G)$. All constant functions $V(G) \rightarrow \mathbb{R}$ are harmonic Dirichlet functions.

For vertices $x$ and $y$ of a graph $G$ let $\delta(x, y)$ denote their distance in $G$. A wobbling is a map $f: V(G) \rightarrow V(G)$ such that $\delta(x, f(x))$ for $x \in V(G)$ is bounded. The map $f: V(G) \rightarrow V\left(G^{\prime}\right)$ is called a quasi-isometry from $G$ to $G^{\prime}$ if there exists a positive number $k$ - the distortion of $f$-such that for vertices $x$ and $y$ in $V(G)$ one has

$$
\frac{1}{k} \delta(x, y)-1<\delta(f(x), f(y)) \leq k \delta(x, y),
$$

and for every vertex $x \in V\left(G^{\prime}\right)$ there exists $y \in V(G)$ with $\delta(x, f(y))<k$. A quasi-inverse of a quasi-isometry $f$ from $G$ to $G^{\prime}$ is a quasi-isometry $g$ from $G^{\prime}$ to $G$ such that $f \circ g$ and $g \circ f$ are wobblings.

Note that for a quasi-isometry $f$ of distortion $k$ one can take a quasi-inverse of distortion $2 k^{2}$.

When speaking of subgraphs of a graph we always mean a connected full subgraph with at least one edge. Let $G_{0}$ be a subgraph of a graph $G$. By $\sigma\left(G_{0}\right)$ we denote the set of vertices of $G_{0}$ that have neighbors in $G$ outside $G_{0}$. We call $G$ amenable if inf $\left|\sigma\left(G_{0}\right)\right| /\left|V\left(G_{0}\right)\right|=0$, where the infimum is taken for finite subgraphs $G_{0}$. We call the sequence $\left(G_{i}\right)$ of finite subgraphs of a graph $G$ a Følner sequence if $\left|\sigma\left(G_{i}\right)\right| /\left|V\left(G_{i}\right)\right|$ tends to 0 as $k$ tends to infinity.

\section{An EXAMPLE}

In this section we construct a roughly transitive amenable graph that is not quasi-isometric to a vertex transitive graph. Let $G$ be a simply connected nilpotent Lie group. Then a net $N$ in $G$ is a roughly transitive amenable graph. Here the vertices of the net $N$ form a maximal subset of $G$ with minimum distance 1 ; the edges connect vertices of distance at most 3 . If $G$ has a co-compact lattice, then of course $N$ is quasi-isometric to the lattice, that is a vertex transitive graph, so the fact that any harmonic Dirichlet function on $N$ is constant follows from Soardi's Theorem [9] on the quasi-isometry invariance of the existence of a non-constant harmonic Dirichlet function. Note, however, that according to Malcev's Theorem [4] $G$ has a co-compact lattice if and only if the Lie-algebra of $G$ is rational i.e. has a base with rational structure constants. There are uncountable pairwise nonisomorphic non-rational Lie-algebras [8]. 
Proposition 2. There exist a simply connected nilpotent Lie group $G$ and a net $N$ of $G$ such that $N$ is not quasi-isometric to any vertex transitive graph.

Proof. Let $\mathcal{G}$ be the graded two-step nilpotent Lie algebra $N(d)$ in [8], where $d$ is irrational. This algebra is non-rational. Let $G$ be the associated simply connected nilpotent Lie group and let $N$ be a net of $G$. Suppose that $N$ is quasi-isometric to a vertex transitive graph $M$. Then $M$ has polynomial growth so by the famous result of Trofimov [11], $M$ is quasi-isometric to the Cayley graph of a discrete nilpotent group $\Gamma$. Let $H$ be the Malcev-completion of $\Gamma$. Then $H$ is a nilpotent Lie group which contains $\Gamma$ as a co-compact lattice. Moreover, the nilpotent Lie groups $G$ and $H$ are quasi-isometric. Now, we recall Pansu's result [6] (we thank Martin Bridson for calling our attention to this beautiful theorem): If two nilpotent Lie groups are quasi-isometric, then their associated graded Lie algebras are in fact isomorphic. The Lie algebra of $H$ is rational by Malcev's Theorem [4. The associated graded Lie algebra of a rational Lie algebra is obviously rational as well. On the other hand the Lie algebra of $G$ is just $N(d)$, which is itself graded. Thus $G$ and $H$ cannot be quasi-isometric. This proves our proposition.

It would be interesting to characterize roughly transitive graphs of polynomial growth up to quasi-isometry the same way as Trofimov did in the vertex transitive case.

\section{The RESUlt}

We borrow some notations from [1. The support $\operatorname{supp}(v)$ of a real-valued function $v$ is the subset of the domain where $v$ is not zero. For a graph $G$ we define $\star(G)$ to be the closure in $l_{2}(G)$ of the functions $d v$, where $v: V(G) \rightarrow \mathbb{R}$ has finite support. Let $\diamond(G)$ be the closure in $l_{2}(G)$ of the flows with finite support.

Notice that we always have $d l_{2}(V(G)) \subseteq \star(G)$. The following lemma is well known. We prove it to be self contained.

Lemma 3. Let $G$ be any graph. We have the following orthogonal decomposition:

$$
l_{2}(G)=\star(G)+\diamond(G)+d H D(G) .
$$

The flows constitute the subspace $\diamond(G)+d H D(G)$, and $d D(G)=\star(G)+d H D(G)$.

Proof. First note that if $v \in D(G)$ and $u$ is a flow with finite support, then we have $\langle d v, u\rangle=\left\langle d v_{0}, u\right\rangle=\left\langle v_{0}, d^{*} u\right\rangle=0$, where $v_{0}$ is $v$ restricted to the finite support consisting of the endpoints of the oriented edges in the support of $u$. Thus $d D(G)$ is orthogonal to $\diamond(G)$. To see that they are orthogonal complements consider any function $u$ orthogonal to $\diamond(G)$. Fix a vertex $x_{0} \in V(G)$ and define $v: V(G) \rightarrow \mathbb{R}$ by $v\left(x_{n}\right)=\sum_{i=1}^{n} u\left(x_{i-1}, x_{i}\right)$ for any path $\left(x_{0}, \ldots, x_{n}\right)(n \geq 0)$ in $G$. The orthogonality proves that $v$ is well defined. We have $d v=u$, thus $d \in D(G)$ as required.

Next we claim that the orthogonal complement of $\star(G)$ consists of all the flows. Indeed a function $u \in l_{2}(G)$ belongs to this complement if and only if $\langle d v, u\rangle=$ $\left\langle v, d^{*} u\right\rangle=0$ for every $v: V(G) \rightarrow \mathbb{R}$ with a finite support. This is satisfied if and only if $d^{*} u=0$.

To finish the proof of the lemma notice that the orthogonal complement of $\star(G)$ contains $\diamond(G)$ and the intersection of the orthogonal complements of $\star(G)$ and $\diamond(G)$ are flows in $d D(G)$, and by definition, this is $d H D(G)$. 
Let $G_{0}$ be a finite subgraph of $G$ and $L$ a closed subspace of $l_{2}(G)$. We define

$$
\operatorname{dim}_{G_{0}}(L)=\frac{\sum_{e \in \vec{E}\left(G_{0}\right)}\left\langle P_{L} e, e\right\rangle}{\left|\vec{E}\left(G_{0}\right)\right|},
$$

where $P_{L}$ is the orthogonal projection to $L$ and the oriented edge $e$ is identified with the element of $l_{2}(G)$ mapping $e$ to $1, \bar{e}$ to -1 and all other oriented edges to 0 .

Claim 4. Let $G_{0}$ be a finite subgraph of a graph $G$. We have $\operatorname{dim}_{G_{0}}\left(l_{2}(G)\right)=$ 1. For orthogonal closed subspaces $L$ and $L^{\prime}$ of $l_{2}(G)$ we have $\operatorname{dim}_{G_{0}}\left(L+L^{\prime}\right)=$ $\operatorname{dim}_{G_{0}}(L)+\operatorname{dim}_{G_{0}}\left(L^{\prime}\right)$. For closed subspaces $L \subseteq L^{\prime}$ one has $0 \leq \operatorname{dim}_{G_{0}}(L) \leq$ $\operatorname{dim}_{G_{0}}\left(L^{\prime}\right)$. If the support of all $u \in L$ is contained in $\vec{E}\left(G_{0}\right)$, then $\operatorname{dim}_{G_{0}}(L)=$ $\operatorname{dim}(L) /\left|E\left(G_{0}\right)\right|$.

Proof. The first statement follows from noting that $P_{l_{2}(G)}$ is the identity. The second statement follows from the equality $P_{L+L^{\prime}}=P_{L}+P_{L^{\prime}}$ for orthogonal subspaces $L$ and $L^{\prime}$. The non-negativity is trivial and implies the monotonicity. For the last statement note that we can work in $l_{2}\left(G_{0}\right)$. Half of the vectors $e \in \vec{E}\left(G_{0}\right)$ (taking one of the pairs $e$ and $\bar{e})$ form an orthonormal basis of $l_{2}\left(G_{0}\right)$, and $\left|E\left(G_{0}\right)\right| \operatorname{dim}_{G_{0}}(L)$ is the trace of $P_{L}$ restricted to $l_{2}\left(G_{0}\right)$ computed in this basis.

Lemma 5. If $G_{0}$ is a finite subgraph of the graph $G$, then $\operatorname{dim}_{G_{0}}(\star(G)+\diamond(G)) \geq$ $1-\left|\sigma\left(G_{0}\right)\right| /\left|E\left(G_{0}\right)\right|$.

Proof. First note that $d H D\left(G_{0}\right)=0$ since every flow on the finite graph $G_{0}$ has finite support. So by Lemma 3 we have $l_{2}\left(G_{0}\right)=\star\left(G_{0}\right)+\diamond\left(G_{0}\right)$.

We identify functions $u \in l_{2}\left(G_{0}\right)$ and $v \in D\left(G_{0}\right)$ with their extension in $l_{2}(G)$ (respectively $D(G)$ ) that is zero outside the original domain. We have to distinguish two differential operators: $d_{G_{0}}: D\left(G_{0}\right) \rightarrow l_{2}\left(G_{0}\right)$ is not the restriction of $d$ : $D(G) \rightarrow l_{2}(G)$ as $d\left(D\left(G_{0}\right)\right) \nsubseteq l_{2}\left(G_{0}\right)$ unless $G=G_{0}$. But using the formula for $d^{*}$ one sees that $d_{G_{0}}^{*}$ is the restriction of $d^{*}$. Thus $\diamond\left(G_{0}\right) \subseteq \diamond(G)$. Let $D_{1}=\{v \in$ $\left.D(G) \mid \operatorname{supp}(v) \subseteq \sigma\left(G_{0}\right)\right\}$ and $D_{2}=\left\{v \in D(G) \mid \operatorname{supp}(v) \subseteq V\left(G_{0}\right) \backslash \sigma\left(G_{0}\right)\right\}$. Clearly, $D\left(G_{0}\right)=D_{1}+D_{2}$ and $\star\left(G_{0}\right)=d_{G_{0}} D_{1}+d_{G_{0}} D_{2}$. Notice that $d$ and $d_{G_{0}}$ are identical in $D_{2}$, thus $d_{G_{0}} D_{2}=d D_{2} \subseteq \star(G)$.

Let $L=d D_{2}+\diamond\left(G_{0}\right)$. We have $d_{G_{0}} D_{1}+L=\star\left(G_{0}\right)+\diamond\left(G_{0}\right)=l_{2}\left(G_{0}\right)$, thus $\operatorname{dim}(L) \geq \operatorname{dim}\left(l_{2}\left(G_{0}\right)\right)-\operatorname{dim}\left(d_{G_{0}} D_{1}\right) \geq\left|E\left(G_{0}\right)\right|-\operatorname{dim}\left(D_{1}\right) \geq\left|E\left(G_{0}\right)\right|-\left|\sigma\left(G_{0}\right)\right|$.

We have $L \subseteq(\star(G)+\diamond(G)) \cap l_{2}\left(G_{0}\right)$, thus by Claim $4 \operatorname{dim}_{G_{0}}(\star(G)+\diamond(G)) \geq$ $\operatorname{dim}_{G_{0}}(L)=\operatorname{dim}(L) /\left|E\left(G_{0}\right)\right| \geq 1-\left|\sigma\left(G_{0}\right)\right| /\left|E\left(G_{0}\right)\right|$ as claimed.

Corollary 6. For a Følner sequence $\left(G_{i}\right)$ of finite subgraphs of a graph $G$ we have $\operatorname{dim}_{G_{i}}(d H D(G))$ tends to zero as $i$ tends to infinity.

Proof. By Lemma 3. Claim 4 and Lemma [5 we have $0 \leq \operatorname{dim}_{G_{i}}(d H D(G)) \leq$ $\left|\sigma\left(G_{i}\right)\right| /\left|E\left(G_{i}\right)\right|$. Here $\left|\sigma\left(G_{i}\right)\right| /\left|E\left(G_{i}\right)\right|$ tends to zero as $\left(G_{i}\right)$ is a Følner sequence. Note that for traditional reasons we used $\left|\sigma\left(G_{i}\right)\right| /\left|V\left(G_{i}\right)\right|$ in the definition of Følner sequences, but since $G_{i}$ is connected and has bounded degree $\left|V\left(G_{i}\right)\right|$ and $\left|E\left(G_{i}\right)\right|$ are proportional.

Corollary [indicates that the harmonic Dirichlet functions on an amenable graph form a "small" subspace. It does not, however, imply that $d H D(G)=0$; this is false for some amenable graphs. Our goal is to prove that if non-constant harmonic 
functions exist on a roughly transitive graph, then they form a "large" subspace contradicting Corollary 6. This is immediate for transitive graphs: with any harmonic Dirichlet function all its translates are harmonic. The case of roughly transitive graphs require more care. We study next how quasi-isometries act on Dirichlet functions and on $d H D(G)$.

Let $G_{1}$ and $G_{2}$ be graphs. For a map $f: V\left(G_{1}\right) \rightarrow V\left(G_{2}\right)$ we define the function $f^{*}: v \mapsto f \circ v$ on the functions $v: V\left(G_{2}\right) \rightarrow \mathbb{R}$. Let $A$ be a subset of the vertices of a graph $G$. For $k>0$ we define the $k$-neighborhood $C_{k}(A)$ of $A$ to be $\{x \in V(G) \mid \exists y \in A: \delta(x, y) \leq k\}$. We define $\chi_{A}: \vec{E}(G) \rightarrow \mathbb{R}$ to be the characteristic function of the oriented edges $(x, y) \in \vec{E}(G)$ with $x \in A$ and $y \in A$.

Lemma 7. For a quasi-isometry $f$ from a graph $G_{1}$ to a graph $G_{2}$ we have that $f^{*}\left(D\left(G_{2}\right)\right) \subseteq D\left(G_{1}\right)$. Furthermore there is a constant $c$ depending on the distortion $k$ of $f$ and the maximum degree of $G_{1}$ such that $\left|d f^{*} v\right| \leq c|d v|$ and $\left|d f^{*} v \cdot \chi_{A}\right| \leq$ $c\left|d v \cdot \chi_{B}\right|$ for any $v \in D\left(G_{2}\right)$ and $A \subseteq V\left(G_{1}\right)$ if $B=C_{k}(f(A))$.

Proof. Let $f$ be as in the lemma. We fix a path $f(x)=x_{0}^{e}, x_{1}^{e}, \ldots, x_{k_{e}}^{e}=f(y)$ in $G_{2}$ of length $0 \leq k_{e} \leq k$ for each oriented edge $e=(x, y) \in \vec{E}\left(G_{1}\right)$. For any $v \in D\left(G_{2}\right)$ we have

$$
\left|d f^{*} v\right|^{2}=1 / 2 \sum_{(x, y) \in \vec{E}\left(G_{1}\right)}(v(f(y))-v(f(x)))^{2} \leq k / 2 \sum_{e \in \vec{E}\left(G_{1}\right)} \sum_{i=1}^{k_{e}}\left(v\left(x_{i}^{e}\right)-v\left(x_{i-1}^{e}\right)\right)^{2} .
$$

The summands in this last expression all appear in the summation $|d v|^{2}=$ $1 / 2 \sum_{(x, y) \in \vec{E}\left(G_{2}\right)}(v(y)-v(x))^{2}$; thus for the first statement we only have to limit the multiplicity of a summand $(v(y)-v(x))^{2}$ in the first sum for any $(x, y) \in \vec{E}\left(G_{2}\right)$. This is the number of oriented edges in $\vec{E}\left(G_{1}\right)$ such that the corresponding (oriented) path in $G_{2}$ contains $(x, y)$. As the endpoints of these edges form a subset in $V\left(G_{1}\right)$ of maximum distance at most $2 k^{2}$, this multiplicity can be bounded in terms of $k$ and the maximum degree of $G_{1}$.

For the last statement notice that if an edge is spanned by a subset $A \subseteq V\left(G_{1}\right)$, then the corresponding path is within the set $B=C_{k}(f(A))$.

Lemma 8. For a wobbling $f$ of a graph $G$ we have $f^{*} v-v \in l_{2}(V(G))$ for every $v \in D(G)$.

Proof. For a vertex $x \in V(G)$ take a path $x=x_{0}, x_{1}, \ldots, x_{k}=f(x)$ in $G$. We have $\left(\left(f^{*} v-v\right)(x)\right)^{2} \leq k \sum_{i=1}^{k}\left(v\left(x_{i}\right)-v\left(x_{i-1}\right)\right)^{2}$. Thus to bound the $l_{2}$ norm of $f^{*} v-v$ in terms of $|d v|$ it is enough to note that we can choose the paths with bounded length and every edge appears in a bounded number of paths. $S\}$.

For a function $f: A \rightarrow B$ and $S \subseteq B$ we use $f^{-1}(S)$ to denote $\{x \in A \mid f(x) \in$

Lemma 9. For a graph $G$ we have $d H D(G) \cong D(G) / d^{-1}(\star(G))$. For a quasiisometry $f$ from $G_{1}$ to $G_{2} f^{*}$ induces an isomorphism between $D\left(G_{2}\right) / d^{-1}\left(\star\left(G_{2}\right)\right)$ and $D\left(G_{1}\right) / d^{-1}\left(\star\left(G_{1}\right)\right)$ thus between $d H D\left(G_{2}\right)$ and $d H D\left(G_{1}\right)$. For quasi-inverses $f$ and $g$ the functions $f^{*}$ and $g^{*}$ induce inverse isomorphisms.

Proof. The $d D(G)=\star(G)+d H D(G)$ claim of Lemma 3 proves the first statement.

Let $f$ be as in the lemma and $v \in d^{-1}\left(\star\left(G_{2}\right)\right)$. Then there exist functions $v_{i} \in D\left(G_{2}\right)$ with finite support such that $d v_{i}$ tend to $d v$ in norm. By Lemma 7 
$d f^{*} v_{i}$ tend to $d f^{*} v$, and as the functions $f^{*} v_{i}$ also have finite support $d f^{*} v \in \star\left(G_{1}\right)$. Thus $f^{*}$ maps $D\left(G_{2}\right) / d^{-1}\left(\star\left(G_{2}\right)\right)$ linearly to $D\left(G_{1}\right) / d^{-1}\left(\star\left(G_{1}\right)\right)$.

To see that this map is an isomorphism take a quasi-inverse $g$ of the quasiisometry $f$. For any $v \in D\left(G_{2}\right)$ we have by Lemma 8 that $g^{*} f^{*} v-v \in l_{2}\left(V\left(G_{2}\right)\right) \subseteq$ $d^{-1}\left(\star\left(G_{2}\right)\right)$. For $v \in D\left(G_{1}\right)$ we similarly have $f^{*} g^{*} v-v \in d^{-1}\left(\star\left(G_{1}\right)\right)$. Thus the maps between $D\left(G_{2}\right) / d^{-1}\left(\star\left(G_{2}\right)\right)$ and $D\left(G_{1}\right) / d^{-1}\left(\star\left(G_{1}\right)\right)$ induced by $f^{*}$ and $g^{*}$ are inverses of each other.

Lemma 10. Let the graph $G$ and the positive numbers $k$ and $\Delta$ be given. If $d H D(G) \neq 0$, then there exist a finite set $A \subseteq V(G)$ and a number $\epsilon>0$ with the following property. For any quasi-isometry $f$ of distortion at most $k$ to $G$ from a graph $G^{\prime}$ of maximum degree at most $\Delta$ one has a function $w \in H D\left(G^{\prime}\right)$ with $|d w|=1$ and $\left|d w \cdot \chi_{B}\right|>\epsilon$ for $B=f^{-1}(A)$.

Proof. Choose a non-constant function $v \in H D(G)$. We fix $\epsilon>0$ later and choose $A=C_{2 k+1}\left(A_{0}\right)$ with a finite set $A_{0} \subseteq V(G)$ such that $\left|d v \cdot \chi_{V(G) \backslash A_{0}}\right|<\epsilon$.

By "constant" we mean a quantity depending on $G, v, k$ and $\Delta$ but not on $G^{\prime}$, $f$ or $\epsilon$.

We can take a quasi-inverse $g$ of $f$ of distortion bounded by a constant. By Lemma 9 we have the decomposition $f^{*} v=v^{\prime}+z$ with some $v^{\prime} \in H D\left(G^{\prime}\right)$ and $z \in d^{-1}\left(\star\left(G^{\prime}\right)\right)$. Similarly $g^{*} v^{\prime}=v+t$ with some $t \in d^{-1}(\star(G))$. By Lemma[7we have $0<|d v| \leq\left|d g^{*} v^{\prime}\right| \leq c\left|d v^{\prime}\right|$ with some constant $c$. Thus $\left|d v^{\prime}\right| \geq c_{0}=|d v| / c>0$. We use Lemma 7 for $f^{*}$ to get $|d z| \leq\left|d f^{*} v\right| \leq c^{\prime}|d v|=c_{1}$ with some constants $c^{\prime}$ and $c_{1}$. Now consider $B=f^{-1}(A) \subseteq V\left(G^{\prime}\right)$ and $C=\left(V\left(G^{\prime}\right) \backslash B\right) \cup \sigma(B)$. Notice that $\chi_{C} \geq 1-\chi_{B}$ and $D=C_{k}(f(C))$ is disjoint from $A_{0}$.

Consider the orthogonal decompositions $d v^{\prime}=u_{1}+u_{2}$ with $u_{1}=d v^{\prime} \cdot \chi_{B}$ and $u_{2}=d v^{\prime} \cdot\left(1-\chi_{B}\right)$, and $d z=s_{1}+s_{2}$ with $s_{1}=d z \cdot \chi_{B}$ and $s_{2}=d z \cdot\left(1-\chi_{B}\right)$. By Lemma 7 we have

$$
\left|u_{2}+s_{2}\right|=\left|d f^{*} v \cdot\left(1-\chi_{B}\right)\right| \leq\left|d f^{*} v \cdot \chi_{C}\right| \leq c_{2}\left|d v \cdot \chi_{D}\right| \leq c_{2}\left|d v \cdot \chi_{V(G) \backslash A_{0}}\right|<c_{2} \epsilon
$$

with a constant $c_{2}$. We can write $0=\left\langle d v^{\prime}, d z\right\rangle=\left\langle u_{1}, s_{1}\right\rangle+\left\langle u_{2}, s_{2}\right\rangle$. Here $\left|\left\langle u_{1}, s_{1}\right\rangle\right| \leq$ $\left|u_{1}\right| \cdot\left|s_{1}\right| \leq\left|u_{1}\right| \cdot|d z| \leq c_{1}\left|u_{1}\right|$ and $\left\langle u_{2}, s_{2}\right\rangle=\left\langle u_{2}, u_{2}+s_{2}\right\rangle-\left|u_{2}\right|^{2} \leq\left|u_{2}\right| \cdot \mid u_{2}+$ $\left.s_{2}|-| u_{2}\right|^{2}<-\left|u_{2}\right|\left(\left|u_{2}\right|-c_{2} \epsilon\right)$. We have $\left|u_{2}\right| \geq\left|d v^{\prime}\right|-\left|u_{1}\right| \geq c_{0}-\left|u_{1}\right|$ hence $c_{1}\left|u_{1}\right| \geq\left\langle u_{1}, s_{1}\right\rangle=-\left\langle u_{2}, s_{2}\right\rangle \geq\left|u_{2}\right|\left(\left|u_{2}\right|-c_{2} \epsilon\right) \geq\left(c_{0}-\left|u_{1}\right|\right)\left(c_{0}-c_{2} \epsilon-\left|u_{1}\right|\right)$ if $c_{0}-c_{2} \epsilon-\left|u_{1}\right|>0$.

Notice that we can choose a small enough $\epsilon$ depending on $c_{0}, c_{1}$, and $c_{2}$ such that $\left|u_{1}\right| \leq c_{1} \epsilon$ contradicts our last inequality. For this $\epsilon$ we have $\left|u_{1}\right|>c_{1} \epsilon$. We take $w=v^{\prime} /\left|d v^{\prime}\right| \in H D\left(G^{\prime}\right)$ and notice that $|d w|=1$ and $\left|d w \cdot \chi_{B}\right|=\left|u_{1}\right| /\left|d v^{\prime}\right|>\epsilon$ as $\left|d v^{\prime}\right| \leq\left|d f^{*} v\right| \leq c_{1}$. The choice of $\epsilon$ (and thus of $A$ ) depends only on $G, v, k$ and $\Delta$.

Now we finish the proof of our theorem. The proof is by contradiction. Let $G$ be a roughly transitive amenable graph with $d H D(G) \neq 0$. Let $k$ be the bound on the distortion of the quasi-isometries $f_{x y}$ from $G$ to $G$ mapping $x \in V(G)$ to $y \in V(G)$. Choose the finite set $A \subseteq V(G)$ and the number $\epsilon>0$ for $G, k$ and the maximum degree $\Delta$ of $G$ as claimed in Lemma 10. We fix a vertex $y \in A$ and use the statement of Lemma 10 to obtain a function $w_{x} \in H D(G)$ for each $x \in V(G)$ such that $\left|d w_{x}\right|=1$ and $\left|d w_{x} \cdot \chi_{B_{x}}\right|>\epsilon$ for $B_{x}=f_{x y}^{-1}(A)$. Let $a=\max _{y^{\prime} \in A} \delta\left(y, y^{\prime}\right)$. By the bound on the distortion of $f_{x y}$ we have $\delta\left(x, x^{\prime}\right)<k(a+1)=b$ for any $x \in V(G)$ and $x^{\prime} \in B_{x}$. 
Let $\left(G_{i}\right)$ be a Følner sequence of finite subgraphs of $G$. Let $S_{i}=V\left(G_{i}\right) \backslash$ $C_{b}\left(\sigma\left(G_{i}\right)\right)$. Note that as $b$ is constant and the degree of the vertices is limited, $\left|C_{b}\left(\sigma\left(G_{i}\right)\right)\right|$ is proportional to $\left|\sigma\left(G_{i}\right)\right|$; thus $\left|S_{i}\right| /\left|V\left(G_{i}\right)\right|$ tends to 1 as $i$ tends to infinity. For $x \in S_{i}$ we have $B_{x} \subseteq C_{b}(\{x\}) \subseteq V\left(G_{i}\right)$. Consider the projection $P$ in $l_{2}(G)$ to $d H D(G)$ and let $P_{x}$ the projection to the one dimensional subspace of $d H D(G)$ generated by $d w_{x}$. Recall that we identify an oriented edge $e \in \vec{E}(G)$ with the function in $l_{2}(G)$ mapping $e$ to $1, \bar{e}$ to -1 and everything else to zero. We have $\langle P e, e\rangle \geq\left\langle P_{x} e, e\right\rangle=\left\langle d w_{x}, e\right\rangle^{2}=\left(d w_{x}(e)\right)^{2}$ for every oriented edge $e$ and vertex $x$ in $G$. We can write $\epsilon^{2}<\left|d w_{x} \cdot \chi_{B_{x}}\right|^{2}=1 / 2 \sum_{e \in \vec{E}(G)} \chi_{B_{x}}(e)\left(d w_{x}(e)\right)^{2} \leq$ $1 / 2 \sum_{e \in \vec{E}(G)} \chi_{B_{x}}(e)\langle P e, e\rangle$ for any $x \in V(G)$. By summation we get $2 \epsilon^{2}\left|S_{i}\right| \leq$ $\sum_{e \in \vec{E}(G)}\langle P e, e\rangle \sum_{x \in S_{i}} \chi_{B_{x}}(e)$ for any index $i$. Notice that $\sum_{x \in S_{i}} \chi_{B_{x}}(e)$ is zero for oriented edges $e$ outside $\vec{E}\left(G_{i}\right)$, while it is bounded by a constant $C$ for any $e \in \vec{E}(G)$; one can take $C$ to be the maximum size of the $b$-neighborhood of a single vertex. Thus we have $2 \epsilon^{2}\left|S_{i}\right| / C \leq \sum_{e \in \vec{E}\left(G_{i}\right)}\langle P e, e\rangle=\left|\vec{E}\left(G_{i}\right)\right| \operatorname{dim}_{G_{i}}(d H D(G))$. Consequently, $\operatorname{dim}_{G_{i}}(d H D(G)) \geq \epsilon^{2} / C \cdot\left|S_{i}\right| /\left|E\left(G_{i}\right)\right|$. By Corollary 6 the left-hand side of this last inequality tends to zero as $i$ tends to infinity, but as $\left|S_{i}\right| /\left|V\left(G_{i}\right)\right|$ tends to 1 and $\left|V\left(G_{i}\right)\right|$ is proportional to $E\left(G_{i}\right)$ the right-hand side does not. The contradiction proves the theorem.

We thank the referee for valuable comments.

\section{REFERENCES}

[1] I. Benjamini, R. Lyons, Y. Peres, and O. Schramm, Uniform spanning forests, preprint, 1998.

[2] J. Cheeger and M. Gromov, $L^{2}$-cohomology and group cohomology, Topology 25 (1986), 189-215. MR 87i:58161

[3] G. EleK, On the Cayley graph of an amenable group, Acta Mathematica Hungarica 74 (1997), no. 3, 229-234. MR 98d:46078

[4] A. Malcev, On a class of homogeneous spaces, AMS Translations 39 (1951). MR 12:589e

[5] G. Medolla and P. M. Soardi, Extension of Foster's averaging formula to infinite networks with moderate growth, Math. Z. 219 (1995), 171-185. MR 96g:94031

[6] P. PAnsu, Métriques de Carnot-Carathéodory et quasiisométries des espaces symétriques de rang un, Annals of Math. 129 (1989), 1-60. MR 90e:53058

[7] W. Paschke, An invariant for finitely presented $C G$-modules, Math. Ann. 301 (1995), no. 2, 325-337. MR 96k:20009

[8] J. Scheuneman, Two-Step Nilpotent Lie Algebras, Journal of Algebra 7 October (1967), 152159. MR 36:225

[9] P. M. SoARdi, Rough isometries and Dirichlet finite harmonic functions on graphs, Proc. of the AMS 119 (1993) , 1239-1248. MR 94a:31004

[10] P. M. SoArdi, Potential theory of infinite networks, Lecture notes in Mathematics 1590 (1994). MR 96i:31005

[11] V.I. Trofimov, Graphs with polynomial growth, Math. Sbornik 51 (1985). MR 85m:05041

Rényi Institute of the Hungarian Academy of Sciences, P. O. Box 127, H-1364 BuDAPEST, HUNGARY

E-mail address: elek@math-inst.hu

Rényi Institute of the Hungarian Academy of Sciences, P. O. Box 127, H-1364 BuDAPEST, HUNGARY

E-mail address: tardos@math-inst.hu 